ARTÍCULO DE REFLEXIÓN

\title{
Concepto de cultura en antropología: el cambio cultural y social
}

Concept of culture in anthropology: cultural and social change

\author{
William Oswaldo Aparicio Gómez ${ }^{1}$ \\ $E d \& T I C$
}

Recibido: 08.11.2020

Aceptado: 22.11.2020

\section{Resumen}

El modelo de cultura sirvió para mostrar cómo el hombre podría simultáneamente ser un individuo, único en el orden biológico y psicológico y ser, al mismo tiempo, fundamentalmente social, viviendo y pensando en función de su grupo de acuerdo con concepciones compartidas gracias a su capacidad de comunicación simbólica. Los conceptos de contacto cultural, de resistencia cultural, de choque cultural, de aculturación, de cambio social y cultural, y más tarde de cambio social aparecen en el campo de la Antropología en la segunda mitad del siglo XIX, en el marco de la expansión capitalista mundial.

Palabras clave: Ciencias de la Educación, Filosofía, Ética, Antropología, Naturaleza humana, Ontología, Humanismo.

\footnotetext{
${ }^{1}$ waparicio@editic.net https://orcid.org/0000-0002-8178-1253
} 


\begin{abstract}
The culture model served to show how man could simultaneously be an individual, unique in the biological and psychological order and be, at the same time, fundamentally social, living and thinking according to his group according to shared conceptions thanks to his capacity symbolic communication. The concepts of cultural contact, cultural resistance, culture shock, acculturation, social and cultural change, and later social change appear in the field of Anthropology in the second half of the 19th century, within the framework of expansion world capitalist.
\end{abstract}

Keywords: Educational Sciences, Philosophy, Ethics, Anthropology, Human Nature, Ontology, Humanism.

\title{
1. Concepto de cultura en antropología
}

El proceso de construcción del concepto de cultura ha sido lento (Colombres, 1985). Reunía varios aspectos: universalidad; relativismo; énfasis en el aspecto estructurado de la vida humana; producto de la actividad colectiva del hombre. Desde el inicio se estableció una diferencia entre la cultura y sus portadores (Chomsky et al., 2019). A la estructuración del concepto siguió su generalización, en cuanto fue adaptado por las demás ciencias sociales.

\subsection{Unidad de análisis y exigencia de conocimiento de la totalidad}

Dentro de las ciencias sociales, la exigencia de totalidad es específica de la Antropología (Hume, 1984). Es imposible tener un verdadero conocimiento de la realidad social si no se parte del conocimiento de la totalidad. 


\subsection{Los grandes conceptos totalizadores}

Las ciencias sociales, entre ellas la Antropología, se valen de algunos conceptos centrales: cultura, sociedad, formación socioeconómica, comunidad, que de ninguna manera son sinónimos: cada uno de ellos aparece recortando de un modo especial la realidad que intenta comprender (Lévi-Strauss, 2010).

\subsection{Cultura, un concepto que se construye}

En el concepto de cultura se puede definir una serie de etapas. Se pueden distinguir:

. El concepto de cultura en el Iluminismo: Una de las características más sobresalientes del pensamiento del iluminismo es la convicción en la fuerza modificadora de la razón, la creencia en el progreso, la exigencia de reemplazo de las instituciones existentes (Colombres, 1985). A lo largo del siglo XVII se constituirán las raíces de los dos usos del concepto de cultura, que continuarán en vivencia hasta nuestros días: lo que Stocking denomina las alternativas humanistas (aquí el humanista estaba ligado al pensamiento iluminista: etnocéntrico) y antropológica (se centra en la idea del relativismo que afirma la validez por igual de los valores y costumbres de todos los pueblos; el interés que por las condiciones que aseguran el mantenimiento del sistema; la idea de que hablamos de una pluralidad de culturas igualmente organizadas para responder a todos los ámbitos de los requerimientos de la vida humana).

. El concepto de cultura en el siglo XIX: Alemania: Hasta que con Tylor se inicie en 1871 la historia oficial del concepto de cultura como idea central de la Antropología, éste se va imponiendo en lengua alemana. El desarrollo alemán hace que participe del desarrollo de la civilización europea como nacionalidad, no como nación unificada; pero su logro era la cultura y el pueblo alemán aparecía entendido como comunidad cultural antes que como comunidad política. 
. El concepto de cultura, en el marco del modelo antropológico clásico: La influencia de Boas es determinante para este nuevo concepto de cultura. La cultura singular de los evolucionistas se convirtió en las culturas plural de la etnología (Boivin et al., 2004). Boas transmitió a sus discípulos la exigencia crítica de estudiar las culturas diversas desde dentro y no desde la óptica del investigador.

. Una teoría científica de la cultura: Un aporte significativo es el de Malinowski, lo que él llama cultura requiere un doble acercamiento: el análisis funcional y el análisis institucional. También en Estados Unidos el concepto de cultura se convirtió en el eje del pensamiento de la antropología. En esa gran entidad que era la antropología cultural era factible diferenciar: interés por la historia cultural, la preocupación por la cultura entendida como herencia social, el interés por el cambio cultural o aculturación y la relación entre cultura y personalidad.

. La realidad de la cultura: Las posiciones realistas conciben la cultura como un atributo del comportamiento social humano (Reis, 2004); la definen en términos de hábitos adquiridos, costumbres e instituciones que no existen independientemente de los grupos concretos a los que se les atribuye.

. La cultura como nivel emergente: Una de las ideas centrales, ligada al núcleo mismo del concepto de cultura, ha sido considerarla como elemento de la existencia humana irreductible a los fenómenos que estudian las ciencias biológicas: a esto se considera nivel emergente. Cultura y sociedad, en este enfoque, son consideradas como entidades superpsíquicas, con leyes propias que no requieren referirse a la naturaleza psicobiológica de los individuos (Panyella, 1962).

\subsection{Crisis del concepto cultura}

Una de las crisis del concepto de cultura ha sido de prolongado trámite: se puede denominar la crisis del concepto de cultura en cuanto éste alude al todo cultural o a la totalidad. Porque, aunque se hable mucho del todo cultural, casi siempre, cuando se habla de la cultura se limita

Volumen 1. Número 2. Julio - Diciembre 2021 
a la selección de normas de conducta que tiende a realizarse de acuerdo con ciertos postulados (valores) básicos para la cultura. A este cambio se han agregado otros: la crisis del contenido relativista del concepto de cultura, la crisis de la relación: una sociedad = una cultura, evidente en el abordaje de las sociedades complejas, la crisis del concepto de socialización y endoculturación desarrollada como parte del concepto de cultura del modelo antropológico clásico (PC, 2001).

Frente al etnocentrismo, ingrediente permanente de la expansión capitalista sobre el resto del mundo, la antropología desarrolló el concepto de relativismo cultural.

\section{Cambio cultural y social: distintos enfoques}

\subsection{Introducción al problema del cambio}

Los conceptos de contacto cultural, de resistencia cultural, de choque cultural, de aculturación, de cambio social y cultural, y más tarde de cambio social aparecen en el campo de la Antropología en la segunda mitad del siglo XIX, en el marco de la expansión capitalista mundial. La aparición del iluminismo; la situación histórica y social del siglo XIX contribuyen a este cambio. La palabra aculturación aparece desde 1880 en los antropólogos norteamericanos, pero es a partir de 1930, sobre todo con el funcionalismo en Inglaterra que estos conceptos y las teorías correspondientes alcanzan su apogeo y desarrollo, ocupando casi por completo el campo de la Antropología (Feixa, 1996).

El colonialismo es percibido por los antropólogos como aculturación o cambio cultural (González Casanova, 2006b). Los estudios sobre aculturación explican el nacimiento de la Antropología aplicada, ésta si bien ha ampliado el campo de acción, de las sociedades etnográficas a las industrializadas, ha permanecido invariable en lo que respecta a su naturaleza misma. 
La relación entre culturas, la aculturación, es una relación asimétrica que está basada en una concepción estratificadora de la sociedad, que supone la dominación de unos sobre otros.

\subsection{Evolucionismo}

Los principales teóricos del evolucionismo lineal, también considerados los padres de la Antropología moderna son Lewis Morgan (1818 - 1881) en Estados Unidos y Edward Burnett Tylor (1834 - 1917) en Inglaterra. El evolucionismo unilateral en Inglaterra se da en el marco histórico y político de la segunda gran expansión europea, caracterizada por la dominación y consecuentemente por la explotación y la violencia. La Antropología evolucionista, habiéndoles asignado una racionalidad a las sociedades primitivas, pretende hacer una reconstrucción esquemática y global de la historia de las sociedades humanas (Casanova, 2007).

Los cuatro aspectos básicos de la sociedad que toma Morgan para estudiar la evolución de la humanidad son: la tecnología, el parentesco, el derecho de propiedad y el sistema político, trabajando en sus comienzos con el material de los indios iroqueses en Estados Unidos (González Casanova, 2006a).

\subsection{Críticas al evolucionismo unilineal}

. Carácter unilineal del cambio sociocultural.

.El cambio sociocultural como producto, esencialmente, de la invención y del descubrimiento.

. La idea de que un patrimonio cultural posee todos sus rasgos en igual medida de avance evolutivo.

. El carácter abstracto e hipotético de sus generalizaciones y teorías.

Volumen 1. Número 2. Julio - Diciembre 2021 
. El etnocentrismo de la Antropología evolucionista inglesa que proyectaba la historia de Europa occidental a todo el ecumene, suponiendo que en todas partes existe la misma secuencia del cambio.

\subsection{Neoevolucionismo}

Leslie White (1900 - 1974); su evolucionismo es unilateral. Su punto de partida es que el hombre utiliza la cultura para satisfacer necesidades y como medio para dominar el hábitat que lo condiciona (Aparicio-Gómez, 2016). En Inglaterra, Elliot Smith y W Perry van a defender esta posición. El presupuesto del difusionismo es que la mayor parte de los pueblos del mundo carecen de capacidad de invención. Para la escuela histórico - cultural, la formación de las diversas culturas se explica a partir de la difusión de unos pocos núcleos o círculos culturales (Césaire, 2019).

\subsection{Materialismo histórico}

El pensamiento marxista va a retomar la teoría de Morgan, sobre todo en el estudio de las sociedades prehistóricas. También desde el pensamiento de Engels vemos que con la esclavitud (que alcanzó su desarrollo máximo bajo la civilización) se realizó la primera escisión de la sociedad en una clase explotadora y una clase explotada. Para Marx la evolución social es dialéctica y no mecanicista. Además, no todas las sociedades tienen que pasar por las mismas etapas en el transcurso de su evolución. El cambio social se opera mediante sucesivas transformaciones en las relaciones de propiedad y de apropiación del excedente social. El agente de cambio es para Marx la lucha de clases, siendo el cambio social esencialmente endógeno.

\subsection{La antropología cultural norteamericana}

A fines del siglo pasado Franz Boas (1858 - 1942) funda en Estados Unidos una escuela que dominó el pensamiento antropológico norteamericano hasta la primera mitad del siglo XX. 
Esta escuela rechazó enérgicamente la concepción unilineal del evolucionismo y el abuso del método comparativo, y contrapuso a la historia conjetural una reconstrucción rigurosamente histórica de los procesos formativos de las instituciones, valorizando el trabajo de los antropólogos. La crítica de la escuela cultural norteamericana al etnocentrismo y a la unidad de la cultura del evolucionismo inglés la condujo a un reconocimiento de la diversidad cultural. El culturalismo norteamericano, relativizando la cultura occidental, pretende superar el asimilacionismo del Iluminismo. El relativismo cultural sostiene que las diferentes culturas tienen sistemas de valores que no deben ser considerados inferiores o superiores a otros; las costumbres, las instituciones, las prácticas, deben ser juzgadas de acuerdo con la cultura a que pertenecen y no según los patrones del antropólogo que las estudia. Este relativismo cae en el psicologismo porque recupera como conocimiento objetivo la idea subjetiva que tienen los individuos de su propia cultura y de los mecanismos que la justifican (Assis, 2014).

\subsection{Escuela funcionalista}

Los máximos representantes del funcionalismo son Bronislaw Malinowski (1884 - 1942) y A. Radcliffe Brown (1881-1955). En la teoría funcionalista, para que exista un orden social tiene que haber determinadas instituciones que, aunque diferentes en cada cultura, cumplan funciones similares: regular las relaciones humanas fundamentales. Estas instituciones se hallan interrelacionadas.

En contra del método histórico y especulativo de los evolucionistas, el funcionalismo propone un estudio en el terreno de las sociedades primitivas, síncrono, aquí y ahora, sin tener en cuenta la historia. En oposición al concepto de supervivencia de los evolucionistas, señala que si persisten en el contexto actual es porque han cambiado de funciones. Para el funcionalismo, las partes o rasgos de una cultura se hallan en relación de interdependencia e integrados funcionalmente. Los antropólogos funcionalistas con su trabajo sobre el terreno empírico y sincrónico constituyen sin lugar a dudas un avance con respecto al evolucionismo y difucionismo en lo referente al cambio. El funcionalismo pone entre paréntesis la situación 
histórica y política en que se realiza la aculturación (Collado-Ruano, 2017). En efecto, la aculturación está desprovista de toda connotación ideológica y política.

\subsection{Los diferentes tipos de colonización: la colonización británica y la colonización francesa}

Generalmente se caracteriza a la colonización inglesa por el gobierno indirecto, siendo en su gran mayoría colonización de explotación. La antropología inglesa genera, desde el evolucionismo unilineal que justifica los primeros contactos racistas hasta el funcionalismo de Malinowski, que justifica indirectamente el conservatismo, el estatismo y el congelamiento de la sociedad colonizada. Para los funcionalistas los estudios antropológicos no son estudios abstractos ni especulativos que respondan al desarrollo interno de la ciencia, sino que tienden a hacer teorías que responden a necesidades concretas: estudios de la organización política tradicional, capacidad de adaptación a la política colonial, etc.

Generalmente se caracteriza a la colonización francesa por el asimilacionismo, gobierno directo, siendo colonización de explotación. En esta colonización la idea central que rige su política es la creencia en la unidad moral del género humano y en el predominio de la razón (Aparicio-Gómez, 2006).

\subsection{Respuestas contra-aculturativas en las sociedades colonizadas: ideologías africanas}

De una manera general, se puede decir que en sus comienzos las más importantes ideologías africanas estaban ligadas al fenómeno de la descolonización política, económica y cultural (Morin, 2012).

. Panafricanismo: Esta ideología nació entre los intelectuales negros de América del Norte a fin del siglo pasado. Su más grande representante era B. du Bois. 
. Negritud: Rechazo de la asimilación cultural francesa, afirmación de los valores culturales negros, la negritud nació en París hacia 1930 en medio de los estudiantes antillanos y africanos. Es una ideología esencialmente intelectualista y culturalista, de ahí sus límites.

. Nacionalismo: Nació en África después de la segunda guerra mundial, en estrecha relación con el panafricanismo, el nacionalismo africano es por una parte uno de sus polos y su concretización a nivel de cada territorio colonizado, por la otra. Por nacionalismo es necesario entender aquí la voluntad del país colonizado en lucha contra el Estado colonial, de obtener la restauración o el reconocimiento de su calidad de Estado soberano y habiéndola obtenido, de mantenerla y de hacerla efectiva.

\section{Antropología aplicada}

La Antropología aplicada no puede considerarse patrimonio del funcionalismo inglés o del culturalismo norteamericano, sino como la conciencia que ambas teorías tienen, en mayor o menor grado y en diferentes momentos de su desarrollo, de un saber práctico (Barnard, 2002). La Antropología aplicada nació de los estudios sobre aculturación y comenzó a formalizarse cuando la aculturación se hizo planificada.

La escuela culturalista norteamericana y su relativismo cultural, sigue un camino que la conduce a la Antropología aplicada, diferente al de Inglaterra.

La Antropología aplicada, a lo largo de su evolución, si bien ha ampliado su campo de acción, de las sociedades simples a las complejas, ha permanecido invariable en lo que respecta a su naturaleza misma: mantiene siempre una relación asimétrica basada en una concepción estratificadora de las sociedades, que supone la dominación de unos sobre otros (Santos, 2003). De acuerdo con esta concepción estratificadora, lo que subyace es la voluntad de asimilar, en el marco de una relación de dominación o dependencia, los menos a los más poderosos, que son quienes fijan las estrategias.

Volumen 1. Número 2. Julio - Diciembre 2021 


\section{Conclusiones}

. En el concepto de cultura se puede definir una serie de etapas.

. Una de las crisis del concepto de cultura ha sido de prolongado trámite.

. El colonialismo es percibido por los antropólogos como aculturación o cambio cultural.

. La relación entre culturas, la aculturación, es una relación asimétrica que está basada en una concepción estratificadora de la sociedad, que supone la dominación de unos sobre otros.

\section{Referencias}

Aparicio-Gómez, Oscar-Yecid (2006). El nexo familia persona en la filosofía personalista (Tesis Doctoral). Barcelona, España: Universidad de Barcelona.

Aparicio-Gómez, Oscar-Yecid (2016). La persona en la Sociedad Digital. En AparicioGómez, Oscar-Yecid (Eds.). El Uso Educativo de las TIC (pp. 251-256). Bogotá, Colombia: Universidad Central. ISBN: 978-958-26-0316-8

Assis, W. F. T. (2014). Do colonialismo à colonialidade: expropriação territorial na periferia do capitalismo. Caderno CRH. https://doi.org/10.1590/s0103-49792014000300011

Barnard, D. A. (2002). Encyclopedia of Social and Cultural Anthropology. In Encyclopedia of Social and Cultural Anthropology. https://doi.org/10.4324/9780203458037

Boivin, M., Rosato, a, \& Arribas, V. (2004). Constructores de otredad. In Buenos Aires: Antropofagia.

Casanova, P. G. (2007). Colonialismo interno (uma redifinição). In A teoria marxista hoje: problemas e perspectiva. 
Césaire, A. (2019). Discurso sobre el colonialismo. Journal of Chemical Information and Modeling.

Chomsky, N., Foucault, M., \& Elders, F. (2019). La naturaleza humana: justicia versus poder. In La naturaleza humana: justicia versus poder. https://doi.org/10.2307/j.ctvndv8qd

Collado-Ruano, J. (2017). Educación y desarrollo sostenible: la creatividad de la naturaleza para innovar en la formación humana. Educación y Educadores. https://doi.org/10.5294/edu.2017.20.2.4

Colombres, R. (1985). Cine, antropología y colonialismo. In Serie antropológica.

Feixa, C. (1996). Antropología de las edades. Ensayos de Antropología Cultural. Homenaje a Claudio Esteva-Fabregat. J. Prat \& A. Martínez (Eds.).

González Casanova, P. (2006a). Colonialismo interno. Una redefinición. La Teoría Marxista Hoy. Problemas y Perspectivas.

González Casanova, P. (2006b). Colonialismo interno. Sociología de La Explotación CLACSO Consejo Latinoamericano de Ciencias Sociales.

Hume, D. (1984). Tratado de la naturaleza humana. In Editorial Orbis.

Lévi-Strauss, C. (2010). Raza y cultura. Lecturas de Antropología Social y Cultural.

Morin, E. (2012). El Método 5: La humanidad de la humanidad. La identidad humana. In Multidiversalidad Mundo Real.

Panyella, A. (1962). Antropología cultural. Boletín Del Seminario de Estudios de Arte y Arqueología: BSAA. 
PC, U. (2001). Antropología de la libertad. In Antropología de la libertad. https://doi.org/10.19083/978-612-318-197-0

Reis, N. I. dos. (2004). Antropología cultural. Horizontes Antropológicos. https://doi.org/10.1590/s0104-71832004000200015

Santos, B. (2003). Entre Próspero e Caliban: colonialismo, pós-colonialismo e interidentidade. Novos Estudos Cebrap. 
REVISTA INTERNACIONAL DE FILOSOFÍA TEÓRICA Y PRÁCTICA

Volumen 1. Número 2. Julio - Diciembre 2021 ISSN: 2745-0333 (En línea) 